\title{
Characterization of readmissions at a Portuguese psychiatric hospital: An analysis over a 21 month period
}

\author{
David Dias Neto \\ Ana Catarina da Silva \\ Hospital Júlio de Matos, Lisboa \\ PORTUGAL
}

\begin{abstract}
Background and Objectives: The readmission rate has been considered as a promising variable to evaluate the efficacy of the mental health system. Considering the lack of literature on the subject in Portugal, it is important to characterize it further in order to validate its meaning as a variable. The aim of this analysis was to characterize readmissions to Júlio de Matos Hospital (JMH), in comparison with the single admissions.

Methods: This analysis was made through the review of the clinical files of patients admitted over a period of 21 months.

Results: Of the 3,225 patients admitted in this period, 1,276 (39.6\%) were readmissions. Furthermore, a readmitted patient was admitted, on average, 2.6 times during the study timeframe.

The results show a reduced social involvement, an increased prior institutionalization and an increased incidence of "Schizophrenic Psychosis" and "Abnormal Personalities" in the sub-group of repeated admissions.

Conclusions: These findings are similar to those obtained in other European countries and strengthen the need for psychosocial and specific interventions at outpatient level. This analysis also shows the value of monitoring the relevant variables to tailor the intervention of the institutions.
\end{abstract}

\section{Introduction}

A significant number of studies have been seeking to understand the high readmission rates of psychiatric patients ${ }^{1,2}$, phenomenon popularized under the name "revolving door".
These studies compare single admission patients with multiple admission patients. The latter are particularly pertinent if we bear in mind that, although they are a minority, these are the patients who spend the most resources ${ }^{3}$. 
The association between the utilization of services and treatment outcome is very complex and may depend upon many factors other than the clinical variables. For example, socio-demographic characteristics of the patient and characteristics of the services are also relevant ${ }^{4}$.

The readmission rate has been found to be associated with severe mental disorder, longer duration of illness, earlier onset of mental illness, worse condition at discharge, co-morbid alcohol or drug problems, present heavy use of inpatient psychiatric resources or frequent previous hospitalization, and poor adherence to medication. Social and demographic factors, such as being unmarried, unemployed, inadequately housed, poorly integrated in the community, involved in the criminal justice system, involuntarily hospitalized at first admission and being unable to gain access to adequate aftercare resources have also been identified as being associated with frequent use of inpatient psychiatric services $^{3,5}$. Institutional aspects, such as bed availability, also seem to be relevant to the readmission phenomenon ${ }^{6}$.

The mental health system is in the process of rapid change. During the last decades the number of beds in psychiatric hospitals has notably decreased and been complemented by day or outpatient services. There has been a reduction in the number of inpatient beds and an increased emphasis on community services ${ }^{7,8}$. Some researchers have explained the increasing number of readmissions as a consequence of de-institutionalization policies ${ }^{9}$ and the development of psychopharmacologic treatments ${ }^{10}$. Given this reduction, it is crucial to develop structures of support in the community and develop programs with the aim of diminishing the number of readmissions ${ }^{7,11}$.

Korkeila and coleagues ${ }^{4}$ observed that patients with a diagnosis of psychosis or personality disorder were admitted twice as often as patients with an organic disorder. They also found that men had more readmissions and were readmitted sooner than women. This was explained by men having more difficulties in coping within the community.

These results allow us to understand what kind of patients are more likely to be readmitted, to predict the clinical and social factors that place them at risk and to identify potential limitations in existing health care delivery systems or specific deficits in available treatment resources ${ }^{12}$. Thus, these aspects can be of great importance for the health system policies ${ }^{3}$ and to the development of interventions with regard to reducing readmissions $^{13,14}$. This may be important in order to reduce superfluous cost associated with readmission.

Readmission rates can also provide an important indicator of the global functioning level of the health system, such as the articulation between inpatient treatment and community interventions. Though readmission rates may not be a suitable indicator of quality of care in psychiatric hospitals ${ }^{15}$, they seem to provide an important tool in planning mental health services.

\section{Aims of the Study}

Given the importance of comprehending this phenomenon and the lack of investigation in this area in Portugal, the present analysis aims to characterize the population with repeated admissions. In order to achieve this goal, a number of socio-demographic and clinical variables were addressed. This study was undertaken at Júlio de Matos Hospital (JMH), which is a psychiatric hospital that covers part of Lisbon and surrounding areas. 


\section{Methods}

The data was gathered in "Psychiatric Clinic VI", which is a short term inpatient ward. This clinic received patients admitted to Júlio de Matos Hospital from the psychiatric emergency room of Curry Cabral Hospital - a general hospital - and occasionally from outpatient services. There was a negligible number of patients who could be directly admitted to acute wards that were unaccounted for in this study. As it was a short length of stay clinic (maximum of seven days), the patients were subsequently admitted to acute wards, referred to another hospital or discharged.

Since the hospital record is done per episode of treatment, the analysis is based on groups of single admissions and readmissions. Therefore, the results of this assessment do not correspond to people but rather to admissions (unless specified otherwise).

The readmission group is constituted by all admissions regarding people that were admitted more than once during the period of the study - 21 months. The single admission group is composed by all admissions regarding people that were admitted only once during that same period. Note that this sub-group also includes people who may have had inpatient treatment before this period. Considering readmissions only during the period of the study, instead of considering all prior admissions as well, has the advantage of excluding the possibility of differences being exclusively due to other factors, such as age.

The analysis addressed a total of 3,252 admissions. All admissions regarding people that were transferred to Curry Cabral Hospital or Santa Maria Hospital (general hospitals) were excluded. This exclusion was due to the fact that most of these patients returned to Júlio de Matos Hospital after the treatment or the evaluation. The inclusion of these admissions could artificially increase the readmission rates. A total of 3,225 admissions were analyzed.

Three groups of variables were accessed: demographic, diagnosis and previous treatments variables. The "diagnosis" variable represents the diagnosis made at the clinic or, if unavailable, the E.R. diagnosis. ICD-9 ${ }^{16}$ is the classification used at the Hospital. Most of these data were gathered from the clinical files, from the patients and from close relatives.

The data were collected from 1 July 2005 to 31 March 2007 (a total of 21 months).

\section{Statistical Analysis}

The statistical analysis was preformed using the Statistical Package for the Social Sciences (SPSS). Given the high $n$, a $p \leq$ 0.001 was considered.

In order to verify the differences in frequency distribution of nominal variables, the Chi Square Test $\left(\chi^{2}\right)$ was used. The difference in mean values of interval variables was accessed using the $T$-Student Test. Both analyses were made in the non-paired samples condition. Given the missing data, a partial $n$ for each variable is presented.

\section{Results}

Of the total 3,225 admissions, 1,276 were readmissions. The general results are presented in Table I.

Table II presents the analysis of sociodemographic variables. The variables in which the frequency varies significantly are 
Table I

General admission data

\begin{tabular}{lc} 
Variable & Value \\
\hline Number of admissions & 3,225 \\
Number of readmissions & 1,276 \\
Percentage of readmissions & $39.6 \%$ \\
Number of $1^{\text {st }}$ admissions $\left(\mathrm{n}=2797^{*}\right)$ & 1,468 \\
Percentage of $1^{\text {st }}$ admissions & $52.5 \%$ \\
Number of admitted patients & 2,440 \\
Number of readmitted patients & 485 \\
Percentage of readmitted patients & $19.9 \%$ \\
Mean admissions per patient & 1.3 \\
Mean admissions per readmitted patient & 2.6 \\
Estimates: & 729.1 \\
Readmissions per year & 277.1 \\
Readmitted patients per year &
\end{tabular}

* Partial n

Table II

Socio-demographic variables per sub-group

\begin{tabular}{clrrrrrrc} 
Variable (n) & & \multicolumn{2}{c}{ Single admissions } & \multicolumn{2}{c}{ Readmissions } & Test & d.f. & P-value \\
\hline Sex & Male & 853 & $(47.4 \%)$ & 570 & $(49.9 \%)$ & $\chi^{2}=1.685$ & 1 & 0.194 \\
$\quad(2,942)$ & Female & 946 & $(52.6 \%)$ & 573 & $(50.1 \%)$ & & & \\
Age & & & & & & & & \\
$\quad(2,928)$ & (years) & $\mathrm{M}=45.0$ & $\mathrm{SD}=15.70$ & $\mathrm{M}=42.2$ & $\mathrm{SD}=14.36$ & $T=4.829$ & $2.575 .7<0.001^{*}$ \\
Nationality & Portuguese & 1,439 & $(89.2 \%)$ & 907 & $(89.2 \%)$ & $\chi^{2}=0.000$ & 1 & 0.983 \\
$\quad(2,631)$ & Other & 175 & $(10.8 \%)$ & 110 & $(10.8 \%)$ & & & \\
Marital & Married/Cohabit** & 623 & $(39.8 \%)$ & 341 & $(34.5 \%)$ & $\chi^{2}=16.158$ & 3 & $0.001^{*}$ \\
Status & Divorced/Separat. & 273 & $(17.4 \%)$ & 166 & $(16.8 \%)$ & & & \\
$(2,553)$ & Single** & 561 & $(35.8 \%)$ & 428 & $(43.4 \%)$ & & & \\
& Widow & 109 & $(7.0 \%)$ & 52 & $(5.3 \%)$ & & & \\
Scholar & (Years) & $\mathrm{M}=7.7$ & $\mathrm{SD}=4.54$ & $\mathrm{M}=7.1$ & $\mathrm{SD}=4.15$ & $T=3.005$ & $1,657.4$ & 0.003 \\
$(1,871)$ & & & & & & & & \\
Professional & Unemployed** & 461 & $(32.5 \%)$ & 385 & $(42.4 \%)$ & $\chi^{2}=25.934$ & 3 & $<0.001^{*}$ \\
Status & Retired & 353 & $(24.9 \%)$ & 214 & $(23.5 \%)$ & & & \\
$(2,328)$ & Employed** & 549 & $(38.7 \%)$ & 277 & $(30.5 \%)$ & & & \\
& Student & 56 & $(3.9 \%)$ & 33 & $(3.6 \%)$ & & & \\
\hline
\end{tabular}

$* p \leq 0.001 ; * *$ Adjusted residuals $\geq 1.96$

marital status and professional status. The mean values presented for age differ significantly from one sub-group to another. Thus, patients who are readmitted to Júlio de Matos Hospital are more often single, unemployed and younger than those admitted only once.
The results presented in Figure 1 point to significant differences in terms of frequency for each sub-group. The major differences point to a higher prevalence of "Schizophrenic Psychosis" and "Abnormal Personalities" in the readmission sub-group. A higher incidence of "Other Psychosis", 


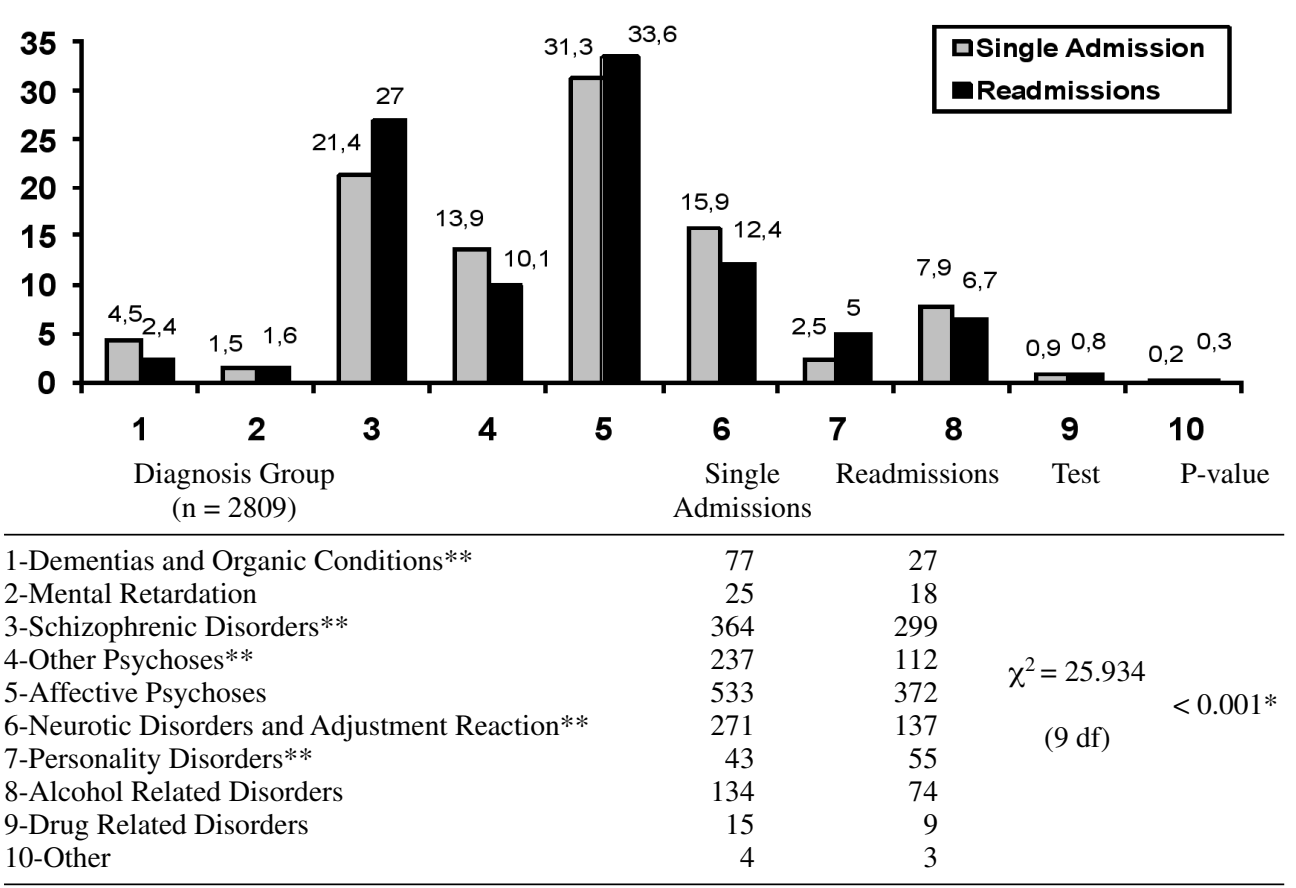

*p $\leq 0.001 ; * *$ Adjusted Residuals $\geq 1.96$

Figure 1. Diagnosis per subgroup (percents and frequencies)

"Neurosis and Adjustment Disorders" and "Dementias and Organic Conditions" is found in the single admission group.

Regarding previous treatments, Table III indicates that average number of total admissions is higher in the readmission subgroup and the mean value for first contact with the hospital is lower in this sub-group.
These results only take into account the period from 1999 onwards, when the hospital created the present clinical filing system.

The results of the variables, Origin $\left(\chi^{2}(1)\right.$ $=3.651, p=0.056)$ and Destination $\left(\chi^{2}(2)=\right.$ $17.153, p=0.002)$, did not achieve statistic significance. However, it should be noted that Destination presents values that are

Table III

Previous treatments per sub-group

\begin{tabular}{llllllll} 
Variable (n) & \multicolumn{2}{c}{ Single admissions } & \multicolumn{2}{c}{ Readmissions } & Test & d.f. & P-value \\
\hline $\begin{array}{l}\text { Total Admissions } \\
(2,791)\end{array}$ & $\mathrm{M}=1.66$ & $\mathrm{SD}=1.65$ & $\mathrm{M}=4.54$ & $\mathrm{SD}=4.70$ & $\mathrm{~T}=-19.460$ & $1,250.3$ & $<0.001^{*}$ \\
$\begin{array}{l}\text { Year of First Contact } \\
(3,222)\end{array}$ & $\mathrm{M}=2004.4$ & $\mathrm{SD}=2.30$ & $\mathrm{M}=2003.1$ & $\mathrm{SD}=2.39$ & $\mathrm{~T}=14.98$ & $2,648.8$ & $<0.001^{*}$ \\
\hline
\end{tabular}


marginally significant, which may suggest a higher number of admissions to acute clinics for readmitted patients when compared to the single admission group.

\section{Discussion}

As mentioned in the Introduction, factors associated with readmissions represent key areas of intervention in reducing the necessity of inpatient treatment. The readmission rate obtained in this study $(39.6 \%)$ is similar to other countries in Europe. We find that readmission rates in other hospitals are close to $40 \%$ after one year, and $50 \%$ after two years ${ }^{15}$. Although this may be a promising rate, it should be noticed that the readmitted patient has twice the number of admissions, has a higher number of previous admissions and had the first contact with the hospital one year sooner than single admission patients. So, there are still some areas in which we can improve intervention.

The characterization of this population may be relevant to establishing alternative interventions to inpatient treatment. The analysis of the demographic variables shows a lower social integration of these patients. They are less likely to have a significant relationship and are most probably unemployed. We can speculate that the disorder may contribute to those conditions but it is also true that this lack of social integration may contribute to relapse. Thus, psychosocial interventions may have an important role in diminishing readmissions.

This analysis also points to two diagnosis sub-groups more associated with readmissions - Schizophrenia and Personality Disorders. If one of the aims of our mental health system is to reduce the "revolving door" phenomenon, then it is important to create specific interventions for these two types of disorders. It should be emphasized that in Schizophrenia successive decompensations may lead to a higher level of functional deficits and that in Personality Disorders inpatient treatment may represent a period of time in which dysfunctional patterns of relationship are reinforced.

Community interventions, such as domiciliary visits, specialized psychotherapeutic interventions and available psychiatric outpatient services, may represent ways of preventing relapse. In this context inpatient care could assume an additional preventive or a palliative functions (e.g. lighten the burden of the families). Accordingly the best outcome variable would be the decrease in days of stay, rather the readmission rate itself.

On the other hand, the higher incidence of "Other Psychosis" and "Neurosis and Adjustment Disorders" in the single admission group may point to the importance of inpatient treatment in some situations. An admission upon a first psychotic episode, a depressive episode or a severe adjustment disorder may have an important prophylactic role.

Finally, present results are similar to those found in the literature. This suggests a phenomenon that may be similar in different cultures.

\section{Limitations and Future Perspectives}

A high number of missing data may have contributed to uncontrolled bias. Additionally, the number of demographic variables gathered was lower than what is desirable 
for a complete assessment. Further study of these dimensions may help to improve the characterization of the population that is continuously readmitted.

Present associations do not necessarily correspond to cause-effect relations in the readmission phenomenon. There is the possibility that third variables may influence the observed differences ${ }^{17}$. This may be minimized in further studies, if other kinds of variables are taken into account.

Variables such as disorder severity or outcome of treatment could be useful in understanding the results of demographic variables and the value of inpatient treatment in the readmission group 5 . Finally, it would be interesting to assess data concerning postdischarge treatment of these patients and the time interval between admissions.

This study was an initial effort to study the theme of readmission in Portugal. It was an endeavor done in the context of clinical practice. Future investigations may add to these observations in order to minimize the effects of the "revolving door" phenomena.

\section{Conclusions}

This study points to the value of investigation in clinical practice. The monitoring of how the service functions and what practices technicians use allows for interesting analyses and the establishment of interventions based on what is observed in a real environment. Considering that sometimes little changes have important impacts on the patients' well being, understanding the relevant variables may constitute an important source of information in mental health practice.

\section{Acknowledgements}

The authors would like to express their appreciation to Fani Lopes who developed and superintended the monitoring of the activity of Psychiatric Clinic VI and to Maria João Carnot, the coordinator of the clinic.

We would also like to express our appreciation for the valuable comments made by the anonymous reviewers

\section{References}

1. Fisher S, Stevens RF. Subgroups of frequent users of an inpatient mental health program at a community hospital in Canada. Psychiatr Serv 1999; 50(2): 244-247.

2. Frazier RS, Casper ES. A comparative study of clinical events as triggers for psychiatric readmission of multiple recidivists. Psychiatr Serv 1998; 49(11): 1423-1425.

3. Bobo WV, Hoge CW, Messina MA, Pavlovcic F, Levandowski D, Grieger, T. Characteristics of repeat users of an inpatient psychiatric service at a large military tertiary care hospital. Milit Med 2004; 169(8): 648-653.

4. Korkeila JA, Lehtinen V, Tuori T, Helenius H. Frequently hospitalised psychiatric patients: a study of predictive factors. Soc Psychiatry Psychiatr Epidemiol 1998; 33(11): 528-534.

5. Hendryx MS, Russo JE, Stegner B, Dyck GD, Ries RK, Roy-Byrne P. Predicting rehospitalization and outpatient services from administration and clinical databases. J Behav Health Serv Res 2003; 30(3): 342-351.

6. Hansson L, Öiesvold T, Göstas G, Kastrup M, Lönnerberg O, Saarento O, et al. The Nordic comparative study on sectorized psychiatry: I. Treated point prevalence and characteristics of the psychiatric. Acta Psychiatr Scand 1995; 91(1): 41-47.

7. Reynolds W, Lauder W, Sharkey S, Maciver S, Veitch $\mathrm{T}$, Cameron D. The effects of a transitional discharge model of psychiatric patients. J Psychiatr Ment Health Nurs 2004; 11(1): 82-88.

8. Thornicroft G, Bebbington P. Deinstitutionalisation from hospital closure to service development. Br J Psychiatry $1989 ; 155: 739-753$. 
9. Kastrup M. The use of a psychiatric register in predicting the outcome 'revolving door patient': A nationwide cohort of first time admitted psychiatric patients. Acta Psychiatr Scand 1987; 76(5): 552-560.

10. Smith CJ, Hanham RQ. Deinstitutionalization of the mentally ill: a time path analysis of the American States, 1955-1975. Soc Sci Med 1981; 15: 361-378.

11. Cougnard A, Parrot M, Grolleau S, Kalmi E, Desage A, Misdrahi D, et al. Pattern of health service utilization and predictors of readmission after a first admission for psychosis: a 2-year follow-up study. Acta Psychiatr Scand 2006; 113(4): 340-349.

12. Kent S, Forgaty M, Yelowwlees P. Heavy utilization of inpatient and outpatient services in a public mental health service. Psychiatr Serv 1995; 46: 1254-1257.

13. Bobier C, Warwick M. Factors associated with readmission to adolescent psychiatric care. Aust N Z J Psychiatry $2005 ; 39(7)$ : 600-606.

14. Mojtabai R, Nicholson RA, Neesmith DH. Factors affecting relapse in patients discharged from a public hos- pital: results from survival analysis. Psychiatr Q 1997; 68(2): 117-129.

15. Lien L. Are readmission rates influenced by how psychiatric services are organized? Nord J Psychiatry 2002; 56(5): 23-28.

16. World Health Organization. The ICD-9 classification of mental and behavioural disorders. Geneva: WHO; 1978.

17. Oiesvold T, Saarento O, Sytema S, Vinding H, Göstas G, Lönnerberg O, et al. Predictors for readmission risk of new patients: the Nordic Comparative Study on Sectorized Psychiatry. Acta Psychiatr Scand 2000; 101(5): 367373.

Address for correspondence: David Manuel Dias Neto Hospital Júlio de Matos. Av. do Brasil, n53. 1749-002 Lisboa E-mail: dmdneto@alunos.fpce.ul Telephone: 00351217917000 Fax: 00351217952989 